\title{
Survival after Salvage Total Laryngectomy: The Influence of Previous Treatment
}

\author{
Ivona Stankovic ${ }^{1}$, Dusan Milisavljevic ${ }^{2}$ and Milan Stankovic ${ }^{2 *}$ \\ ${ }^{1}$ Department of Physical and Mental Rehabilitation, Medical Faculty, Nis, Serbia \\ ${ }^{2}$ Clinic for Otorhinolaryngology, Clinical Center, Nis, Serbia
}

*Corresponding author: Milan Stankovic, Department of Otolaryngology, Bul. Z. Djindjica 52, 18000 Nis, Serbia, Tel: +38118-531-272; Fax: +38118-4531272; E-mail: milanorlstankovic@gmail.com

Received date: June 09, 2016; Accepted date: December 20 2016; Published date: January 02, 2017

Copyright: ( 2017 Stankovic I, et al. This is an open-access article distributed under the terms of the Creative Commons Attribution License, which permits unrestricted use, distribution, and reproduction in any medium, provided the original author and source are credited.

\begin{abstract}
Purpose: To compare the complications and survival rate after different treatment modalities of advanced laryngopharyngeal cancer.
\end{abstract}

Methods: Retrospective study included 619 advanced laryngopharyngeal carcinoma, treated with either primary total laryngectomy (PTL), or salvage (STL) after partial laryngectomy, radio, chemoradiotherapy. Complications and survival rate were documented.

Results: Five years disease free survival rate amounted $60.9 \%$ for PTL, $54.3 \%$ for STL after partial laryngectomy, $50 \%$ for STL after radiotherapy and $43.8 \%$ for STL after chemoradiotherapy. Histologically positive neck was highly significantly associated with worse prognosis, much more than recurrence within larynx.

Conclusion: PTL gives the best survival rate with low complications for advanced laryngopharyngeal squamous cell carcinoma. Complications and survival rate of STL significantly depend on previous treatment of laryngopharyngeal cancer.

Keywords: Laryngopharyngeal cancer; Laryngectomy; Survival; Radiotherapy

\section{Introduction}

During the latest 20 years chemoradiotherapy became popular treatment of laryngeal carcinoma promising preservation of larynx and its function. However, despite multidisciplinary treatment modalities advanced squamous cell carcinoma affecting larynx and laryngopharynx often demands radical surgery, either primary total laryngectomy (PTL), or salvage laryngectomy after failure of previous treatment (STL).

In advanced stages recurrences are frequent, survival is decreased, and quality of life is reduced, leading to bad prognosis. Also, salvage surgery is accompanied by higher complication rate, and worse survival rate [1-4].

Studies that analyse the influence of previous treatment on the survival after STL are scant. So, we conducted a retrospective study to compare the survival rate for STL for recurrences after previous partial laryngectomy, radiotherapy, and chemoradiotherapy.

\section{Methods}

This retrospective study included 665 total laryngectomies performed in the period between 1991 and 2010 for advanced squamous cell carcinoma of the larynx and laryngopharynx. Overall 619 patients were adequately followed up, and their histories were analyzed. PRT was offered to all patients with advanced cancer, and it was undertaken in 442 patients. Patients that refused surgery, or had surgical contraindications were treated by radiotherapy, or chemoradiotherapy (for any nodal affection documented using computed tomography).

Salvage surgery was performed in 35 patients after previous partial laryngectomy, in 78 after initial radiotherapy (60 Gy to $70 \mathrm{~Gy}$ ), and in 64 patients after chemoradiotherapy (cisplatin +5 -fluorouracil). Postoperative clinical examination was performed every 3 months during 5 years. Complications, residual and recurrent disease, and disease free survival rate were documented.

Disease free survival was calculated using the Kaplan-Meier method from the time of initiation of therapy to the time of progression, death, or last follow up. All statistical analyses were performed using SPSS version 12 (SPSS Inc., Chicago, IL, USA). Chi square test was used to investigate the differences between the groups of the patients ( $p$ value less than 0.05 denoted the presence of a statistically significant difference).

\section{Results}

No significant difference was found for analyzed treatment groups for age, sex, smoking and drinking habits, and TNM stage. Patients treated by primary radio and chemoradiotherapy had more comorbidities. Contrary to this, primary total laryngectomy gave significantly less complications (infection, fistula, and difficult swallowing), than for salvage surgery.

Previous chemoradiotherapy caused the highest rate of complications. Nonsurgical primary treatment of laryngopharyngeal localization was accompanied by significantly more complications, than for only laryngeal affection (Tables 1 and 2, Figure 1). 
Citation: Stankovic I, Milisavljevic D, Stankovic M (2017) Survival after Salvage Total Laryngectomy: The Influence of Previous Treatment. J Nucl

Page 2 of 3

Five years disease free survival rate amounted $60.9 \%$ for PTL, $54.3 \%$ for STL after partial laryngectomy, 50.0\% for STL after radiotherapy, and $43.8 \%$ for STL after chemoradiotherapy. Histologically positive neck was highly significantly associated with worse prognosis, much more than recurrence within larynx (Tables 1 and 2, Figure 1).

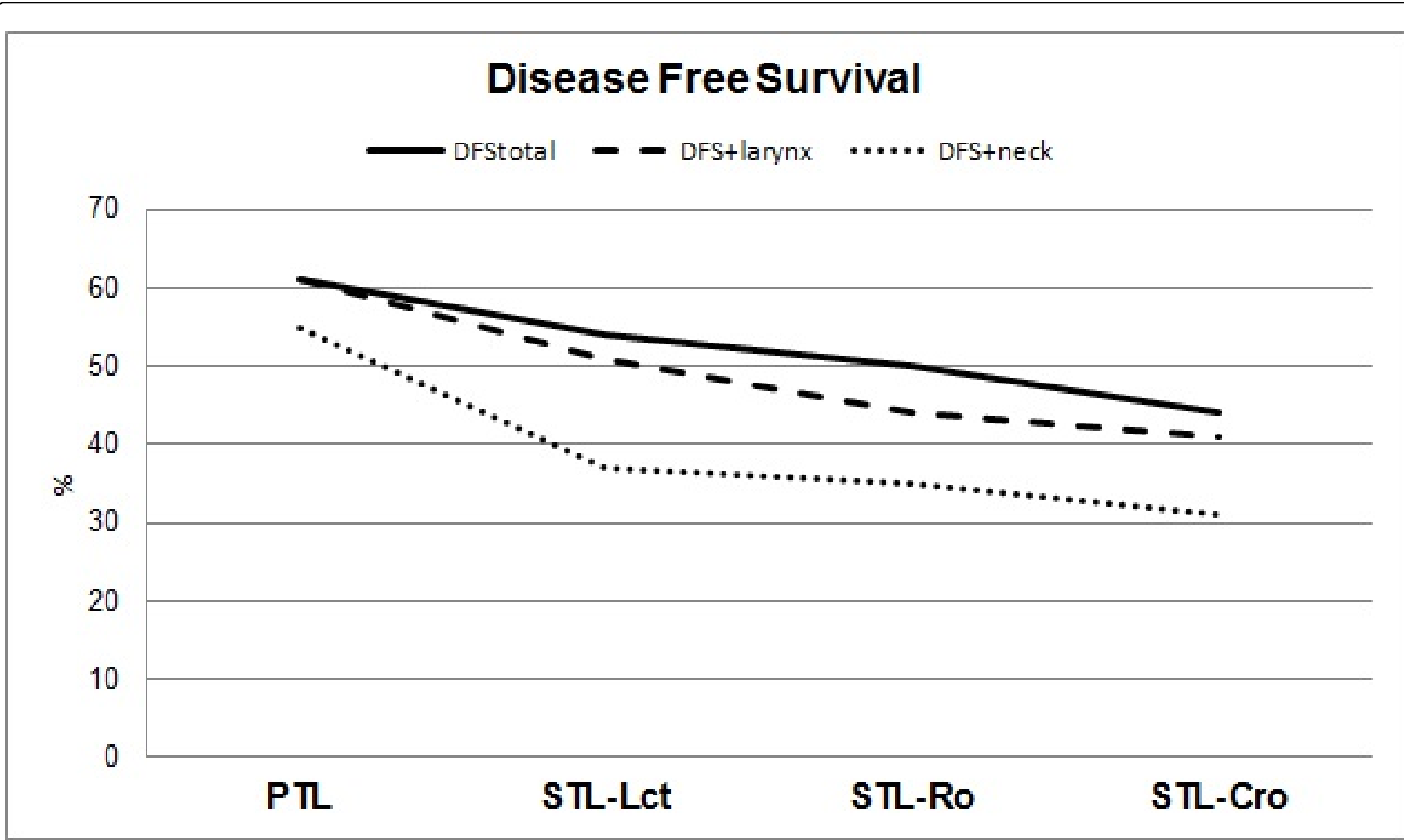

Figure 1: Overall five years' disease free survival rate for advanced laryngeal and laryngopharyngeal carcinoma (histogram). PTL=Primary Total Laryngectomy; STL-Lct=Salvage After Partial Laryngectomy; STL-Ro=Salvage After Radiotherapy; STL-CRo=Salvage After Chemoradiotherapy; DFS Total=Disease Free Survival for All Patients; DFS+Larynx=Disease Free Survival with Recurrence Affecting Larynx; DFS+Neck=Disease Free Survival with Recurrence Affecting Neck.

\begin{tabular}{|c|c|c|c|c|c|c|c|c|}
\hline \multirow[t]{2}{*}{ Parameter } & \multicolumn{2}{|c|}{ PTL (No. 384) } & \multicolumn{2}{|c|}{ STL-Lct (No. 24) } & \multicolumn{2}{|c|}{ STL-Ro (No. 47) } & \multicolumn{2}{|c|}{ STL-CRo (No. 39) } \\
\hline & No. & $\%$ & No. & $\%$ & No. & $\%$ & No. & $\%$ \\
\hline Reconstruction & 0 & 0.0 & 3 & $12.5^{*}$ & 4 & $8.5^{*}$ & 6 & $15.4^{*}$ \\
\hline Infection & 69 & 18.0 & 10 & $41.7^{*}$ & 13 & $27.7^{*}$ & 19 & $48.7^{*}$ \\
\hline Fistula & 55 & 14.3 & 6 & $25.0^{*}$ & 9 & 19.1 & 14 & $35.9^{*}$ \\
\hline Diff. swallow. & 25 & 6.5 & 10 & $41.7^{*}$ & 16 & $34.0^{*}$ & 14 & $35.9^{*}$ \\
\hline DFS total & 238 & 68.0 & 14 & $58.3^{*}$ & 25 & $53.2^{*}$ & 18 & $46.1^{*}$ \\
\hline DFS+Larynx & 238 & 68.0 & 14 & $58.3^{*}$ & 24 & $51.1^{*}$ & 17 & $43.6^{*}$ \\
\hline DFS+Neck & 216 & 56.3 & 10 & $41.7^{*}$ & 19 & $40.4^{*}$ & 15 & $38.5^{*}$ \\
\hline
\end{tabular}

Table 1: Complications and five years disease free survival rate after treatment of advanced laryngeal carcinoma. 


\begin{tabular}{|c|c|c|c|c|c|c|c|c|}
\hline \multirow[t]{2}{*}{ Parameter } & \multicolumn{2}{|c|}{ PTL (No. 58) } & \multicolumn{2}{|c|}{ STL-Lct (No. 11) } & \multicolumn{2}{|c|}{ STL-Ro (No. 31) } & \multicolumn{2}{|c|}{ STL-CRo (No. 25) } \\
\hline & No. & $\%$ & No. & $\%$ & No. & $\%$ & No. & $\%$ \\
\hline Reconstruction & 6 & 10.3 & 6 & $54.5^{*}$ & 10 & $32.3^{*}$ & 11 & $44.0^{*}$ \\
\hline Infection & 12 & 20.7 & 5 & $45.5^{*}$ & 14 & $45.2^{*}$ & 18 & $72.0^{*}$ \\
\hline Fistula & 10 & 17.2 & 4 & $36.4^{*}$ & 9 & 29.0 & 15 & $60.0^{*}$ \\
\hline Diff. swallow. & 6 & 10.3 & 4 & $36.4^{*}$ & 7 & $22.6^{*}$ & 8 & $32.0^{*}$ \\
\hline DFS total & 31 & 53.4 & 5 & $45.5^{*}$ & 14 & $45.2^{*}$ & 10 & $40.0^{*}$ \\
\hline DFS+Larynx & 31 & 53.4 & 4 & $36.4^{*}$ & 10 & $32.2^{*}$ & 9 & $36.0^{*}$ \\
\hline DFS+Neck & 25 & 43.1 & 3 & $27.3^{*}$ & 8 & $25.8^{*}$ & 5 & $20.0^{*}$ \\
\hline
\end{tabular}

Table 2: Complications and five years disease free survival rate after treatment of advanced laryngopharyngeal carcinoma.

\section{Discussion}

Multi-institutional studies in the United States encouraged organ preservation strategy for the management of the primary site in head and neck squamous cell carcinoma. Preservation of larynx means larynx in place, no residual tumor, and also no tracheotomy and no feeding tube. Preservation of function is more important than just anatomic integrity of the larynx [5-8].

Higher survival rates for laryngeal cancer were confirmed after primary laryngectomy, and organ preservation strategy resulted in decrease of global survival for laryngeal cancer from $66 \%$ to $63 \%$ in the last years [1,4]. Radiotherapy for laryngeal cancer produces more frequent fistulas especially when bigger fields and doses were used. Also, chemotherapy was a single factor for complications, such as impaired healing, infection, dehiscence and fistula, with higher rates when radiotherapy is added [9-11].

A systematic review of the literature that included 50 studies with 3292 patients after STL documented overall complication rate in $67.5 \%$, and pharyngocutaneous fistula had incidence of $28.9 \%$ [9-11]. Five years disease fee survival rate in this study depended significantly on: advanced clinical disease stage, tumor localization, early recurrency and previous therapy. Compared to STL, primary radical surgery produced the best survival rates, with fewer complications.

This implies that chemoradiotherapy as initial treatment option for advanced laryngopharyngeal cancer should be offered when surgery is contraindicated, and also knowing complications and survival rate compared to primary surgery. Early diagnosis and better initial radioand chemotherapeutical modalities are needed. The strength of the study is a relatively big number of treated patients, and comparison of PTL and STL, while weakness is its retrospective nature.

\section{Conclusion}

Primary total laryngectomy (PTL) for advanced laryngopharyngeal carcinoma is associated with less complications and better disease free survival rate than salvage surgery (STL). Previous treatment (partial laryngectomy, radio, or chemoradiotherapy) was significant factor for complications and survival rate.

\section{References}

1. Lefebvre JL (2009) Surgery for Laryngopharyngeal SCC in the Era of Organ Preservation. Clin Exp Otorhinolaryngol 2: 159-163.

2. Hoffman HT, Porter K, Karnell LH, Cooper JS, Weber RS, et al. (2006) Laryngeal Cancer in the United States: Changes in Demographics, Patterns of Care, and Survival. Laryngoscope 116: 1-13.

3. Olsen KD (2010) Reexamining the Treatment of Advanced Laryngeal Cancer. Head Neck 32: 1-7.

4. Weber RS, Berkey BA, Forastiere A, Cooper J, Maor M, et al. (2003) Outcome of Salvage Total Laryngectomy following Organ Preservation Therapy. Arch Otolaryngol Head Neck Surg 129: 44-49.

5. Stoeckli SJ, Pawlik AB, Lipp M, Huber A, Schmid S (2000) Salvage Surgery after Failure of Nonsurgical Therapy for Carcinoma of the Larynx and Hypopharynx. Arch Otolaryngol Head Neck Surg 126: 1473-1477.

6. Herranz J, Sarandeses A, Fernandez MF, Barro CV, Vidal JM, et al. (2000) Complications after Laryngectomy in Nonradiated Laryngeal and Hypopharyngeal Carcinomas. Otolaryngol Head Neck Surg 122: 892-898.

7. Cavalot AL, Gervasio CF, Nazionale G, Nazionale G, Albera R, et al. (2000) Pharyngocutaneous Fistula as a Complication of Total Laryngectomy: Rewiev of the Literature and Analysis of Case Records. Otolaryngol Head Neck Surg 123: 587-592.

8. Morgan JE, Breau RL, Suen JY, Hanna EY (2007) Surgical Wound Complications after Intensive Chemoradiotherapy for Advanced Squamous Cell Carcinoma of the Head and Neck. Arch Otolaryngol Head Neck Surg 133: 10-14.

9. Hasan Z, Dwivedi RC, Gunaratne DA, Virk SA, Palme CE, et al. (2006) Systematic review and meta-analysis of the complications of salvage total laryngectomy. Eur J Surg Oncol 43: 42-51.

10. Sandulache VC, Vandelaar LJ, Skinner HD, Cata J, Hutcheson K, et al. (2016) Salvage total laryngectomy after external-beam radiotherapy: A 20-year experience. Head Neck 38: E1962-E1968.

11. Delgado IL, Ayora JR, Brítez OA, Lopez IG, Guirado TM, et al. (2014) Salvage surgery for locoregional recurrences of advanced pharyngolaryngeal squamous cell carcinoma after organ preservation failure. Acta Otorhinolaryngol Ital 34: 382-388. 